\title{
Engineering Gaussian disorder at rough interfaces for light trapping in thin-film solar cells
}

\author{
Piotr Kowalczewski, * Marco Liscidini, and Lucio Claudio Andreani \\ Department of Physics, University of Pavia, Via Bassi 6, Pavia I-27100, Italy \\ *Corresponding author: piotr.kowalczewski@unipv.it
}

Received September 24, 2012; accepted October 5, 2012;

posted October 16, 2012 (Doc. ID 176751); published November 22, 2012

\begin{abstract}
A theoretical study of randomly rough interfaces to obtain light trapping in thin-film silicon solar cells is presented. Roughness is modeled as a surface with Gaussian disorder, described using the root mean square of height and the lateral correlation length as statistical parameters. The model is shown to describe commonly used rough substrates. Rigorous calculations, with short-circuit current density as a figure of merit, lead to an optimization of disorder parameters and to a significant absorption enhancement. The understanding and optimization of disorder is believed to be of general interest for various realizations of thin-film solar cells. (c) 2012 Optical Society of America OCIS codes: $040.5350,050.1950$.
\end{abstract}

Reducing the thickness of a solar cell active layer improves quality of the film and decreases material consumption [1], but the device performance deteriorates due to poor absorption. For this reason, increasing optical thickness of the device by light trapping [2] is a fundamental issue in thin-film solar-cell design. A particularly interesting approach to obtain light trapping is the use of properly optimized rough interfaces [3-8].

In this Letter, we present a theoretical study of a randomly rough Gaussian surface, which is used as a light-trapping interface within a silicon solar-cell structure. Our purpose is twofold: to show that the model of Gaussian disorder gives a good description of actual rough substrates used in real solar cells, and to determine the optimal roughness parameters yielding the highest absorption enhancement in the active layer.

We consider the case of a single rough interface [see Fig. 1(a)] described in real space by the root mean square (RMS) of height $\sigma$ and the correlation length $l_{c}$ [9], which is defined as the distance at which the correlation function $W(|x|)=\exp \left(-x^{2} / l_{c}^{2}\right)$ decreases by $1 / e[10]$. Notice that the average spacing between consecutive maxima/ minima of the rough surface is given by $1.28 \times l_{c}$. The algorithm to generate random surface profiles with given statistical parameters $\sigma$ and $l_{c}$ was derived in [11]:

$$
\begin{aligned}
\xi(x)= & \sigma \sqrt{\frac{2}{\Lambda}} \sum_{m=1}^{\infty} \sqrt{g\left(\frac{2 \pi m}{\Lambda}\right)} \\
& \times\left[\alpha_{2 m-1} \sin \left(\frac{2 \pi m x}{\Lambda}\right)+\alpha_{2 m} \cos \left(\frac{2 \pi m x}{\Lambda}\right)\right],
\end{aligned}
$$

where $\alpha$ is a random number with the normal distribution, $\Lambda$ is the period of the surface profile, and $g(k)=$ $\sqrt{\pi} l_{c} \exp \left(-k^{2} l_{c}^{2} / 4\right)$ is the power spectrum. This Gaussian surface model is known to give a good representation for a wide class of random surfaces [9].

Calculations were done by solving Maxwell equations for unpolarized light using rigorous coupled-wave analysis $[12,13]$. The rough surface profile is discretized in the vertical direction, and we consider a computational cell of length equal to $\Lambda$ in Eq. (1). We use lengths between 10 and $20 \mu \mathrm{m}$, which allow us to neglect the effects of periodicity. Convergence with numbers of plane waves and discretization layers has been carefully checked.

We validate this simple model by describing common rough substrates used for thin-film solar cells. To avoid making a material-dependent comparison, we choose the benchmark situation considered in [5], namely a rough interface between a transparent conductive oxide (TCO) with refractive index $n_{\mathrm{TCO}}=1.915$ and a dielectric medium with specified $n$. In Fig. 2 we show (a) the haze and (b) the angle-resolved scattering (ARS) function calculated with the parameters of two typical substrates: Neuchâtel $\left(\sigma=81 \mathrm{~nm}, \quad l_{c}=140 \mathrm{~nm}\right)$ and Asahi-U $\left(\sigma=35 \mathrm{~nm}, l_{c}=160 \mathrm{~nm}\right)$. These results have been obtained by averaging over 500 surface realizations having the same statistical parameters. Both the haze and the ARS calculated with the Gaussian model are in good agreement with those reported in Fig. 2 of [5], which are obtained by importing the measured topographic profile of real substrates into the finite-difference time-domain calculation (differences in the central dip of the ARS are believed to be due to our use of finer spacing and better resolution for the polar angle $\theta$ ). The agreement is surprisingly good, considering that the present Gaussian model is one-dimensional (1D), while the real substrates are two-dimensional (2D).

Let us now consider the solar cell structure presented in Fig. 1(b). It consists of a $70 \mathrm{~nm}$ thick homogeneous TCO layer $\left(n_{\mathrm{TCO}}=1.65\right.$, nonabsorbing $)$ on a $1 \mu \mathrm{m}$ thick crystalline silicon slab [14], and a silver back reflector [14]. The top layer is used as an antireflection (AR) coating, while the rough interface between the silicon and

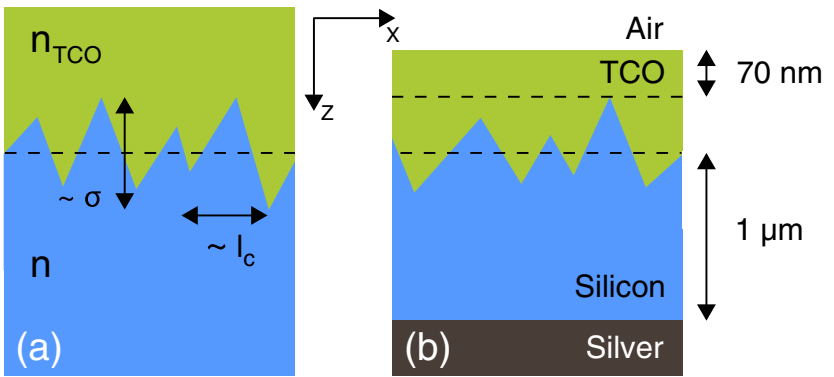

Fig. 1. (Color online) Sketch of (a) rough interface and (b) solar-cell structure under investigation. 

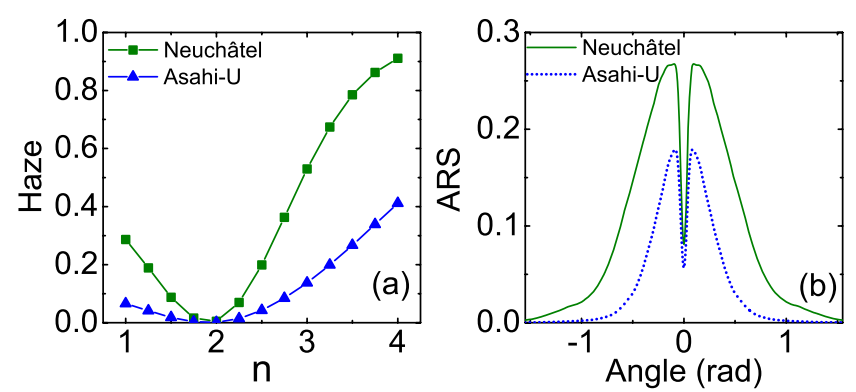

Fig. 2. (Color online) (a) Haze for the interface between two rough TCO substrates $\left(n_{\mathrm{TCO}}=1.915\right)$ and a medium with varying refractive index $n$. Curves are guides to the eye. (b) ARS function for the two interfaces. Substrate parameters: see [5] and text. The wavelength is $\lambda=633 \mathrm{~nm}$.

TCO layers is responsible for light trapping. The choice of c-Si is motivated by recent developments on epitaxy-free fabrication of thin c-Si layers and solar cells [15].

In Fig. 3 we show absorption spectra for $l_{c}=100 \mathrm{~nm}$ and different values of $\sigma$. The curve for $\sigma=0$ corresponds to a flat Si/TCO interface, and it displays FabryPérot oscillations. The other spectra are obtained by averaging over 10 surface realizations. Upon increasing $\sigma$, the oscillations are smeared out by the roughness. This is consistent with previous analyses of, totally or partially, disordered photonic structures [16-19], which all showed that no sharp peaks occur in the presence of disorder. Globally, the disordered structures perform considerably better in both the low-energy and the highenergy ranges, while the flat structure gives higher absorption peaks only in a restricted interval around $2.5 \mathrm{eV}$. Thus, the rough interface achieves both efficient diffraction at low energies and efficient $\mathrm{AR}$ action at high energies.

The crucial figure of merit for a solar cell is the shortcircuit current density. We integrate light absorption over the solar spectrum [20], assuming unity carrier collection efficiency. As in our previous work [21], we take a blackbody spectrum at a temperature of $5800 \mathrm{~K}$ normalized to the standard irradiance of $100 \mathrm{~mW} / \mathrm{cm}^{2}$.

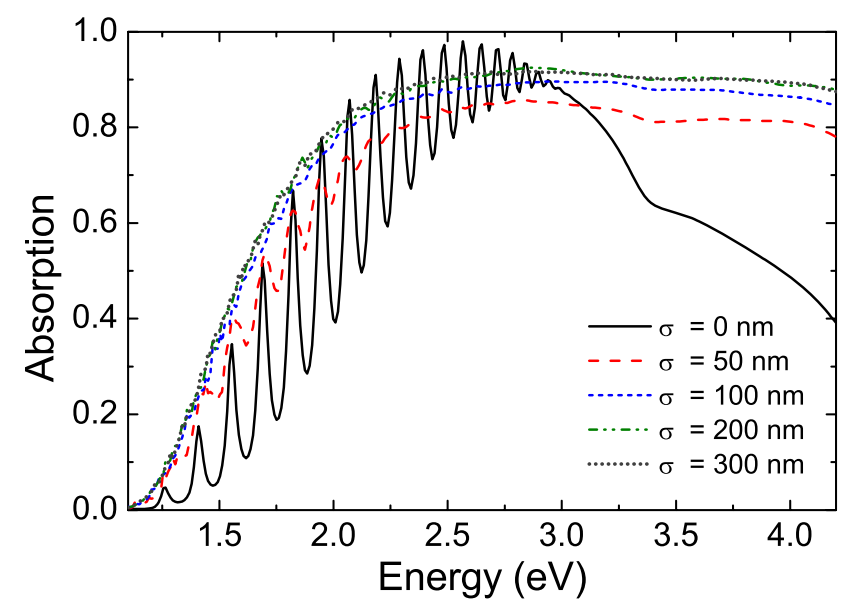

Fig. 3. (Color online) Absorption spectra for structures with $l_{c}=100 \mathrm{~nm}$ and different values of RMS height. The black curve for $\sigma=0$ corresponds to the flat structure.
In Fig. 4 we show $J_{\mathrm{sc}}$ as a function of $\sigma$ for the solar cell structure of Fig. 1(b), assuming $l_{c}=100 \mathrm{~nm}$. For $\sigma$ larger than a few tens of nanometers, $J_{\mathrm{sc}}$ increases and saturates around $21.5 \mathrm{~mA} / \mathrm{cm}^{2}$ for $\sigma$ larger than about $150 \mathrm{~nm}$. The behavior as a function of $\sigma$ is very similar to that calculated for a-Si solar cells $[7,8]$. Again, the 1D Gaussian model captures the same physical behavior that has been previously shown for rough $2 \mathrm{D}$ substrates. To perform a more detailed analysis, the inset in Fig. $\underline{4}$ shows the small- $\sigma$ region calculated for $d_{\mathrm{TCO}}=70 \mathrm{~nm}$ [as in Fig. 1(b)] and $d_{\mathrm{TCO}}=1 \mu \mathrm{m}$. In the latter case $J_{\mathrm{sc}}$ increases quadratically at small $\sigma$, which is reminiscent of the scattering losses in photonic crystal waveguides in the perturbative regime $[\underline{22}, \underline{23}]$. When $d_{\mathrm{TCO}}=70 \mathrm{~nm}$, the short-circuit current decreases from the $\sigma=0$ value. We interpret this behavior as follows: $d_{\mathrm{TCO}}=70 \mathrm{~nm}$ is the optimized width for AR action, but by introducing the roughness the AR property gets partially lost, leading to a decrease of $J_{\mathrm{sc}}$. When $d_{\mathrm{TCO}}=1 \mu \mathrm{m}$, the TCO layer is not optimized as AR coating; thus $J_{\text {sc }}$ starts from a smaller value at $\sigma=0$, but it increases monotonically with $\sigma$. An important message here is that $J_{\mathrm{sc}}$ is independent of the TCO thickness for $\sigma$ larger than a few tens of nanometers.

In Fig. $\underline{5}$ we show the short-circuit current density calculated as a function of $l_{c}$, between 60 and $220 \mathrm{~nm}$, and $\sigma$, between 0 and $300 \mathrm{~nm}$. Each point has been averaged over 10 surface realizations. The dependence on $l_{c}$ at fixed $\sigma$ has a bell-like shape, with a maximum at an optimal value of $l_{c}$ [ [ ]]. This optimal $l_{c}$ is dependent on $\sigma$, increasing from less than $80 \mathrm{~nm}$ at low $\sigma$ to about $160 \mathrm{~nm}$ for $\sigma=300 \mathrm{~nm}$. In Fig. $\underline{5}$ we indicate the position of Neuchâtel and Asahi-U substrates, for the statistical parameters given in [5] (the Jülich substrate, having $l_{c} \sim 1.4 \mu \mathrm{m}$, lies outside our calculation range). This shows that (1) there is margin for improving light trapping by optimizing rough substrates, and (2) analyses for specific solar-cell structures should take into account correlations between the disorder parameters $\sigma$ and $l_{c}$.

While the results of Fig. 2 suggest the qualitative behavior of the short-circuit current density as a function of $\sigma$ and $l_{c}$ to be the same for the present 1D model and for the $2 \mathrm{D}$ interfaces, the values of $J_{\mathrm{sc}}$ are expected to be

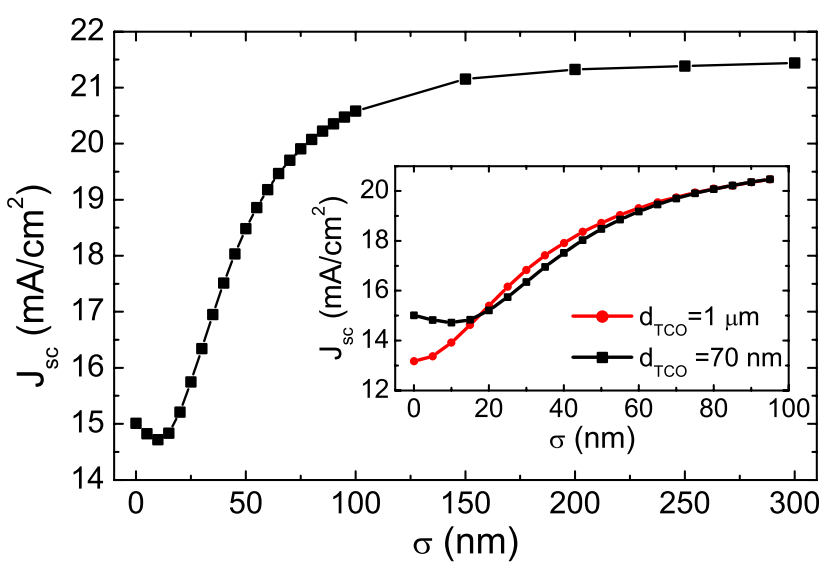

Fig. 4. (Color online) Short-circuit current density as a function of RMS height for $l_{c}=100 \mathrm{~nm}$ and $d_{\mathrm{TCO}}=70 \mathrm{~nm}$. Inset: blow-up of small- $\sigma$ region for $70 \mathrm{~nm}$ and $1 \mu \mathrm{m}$ TCO thickness. 


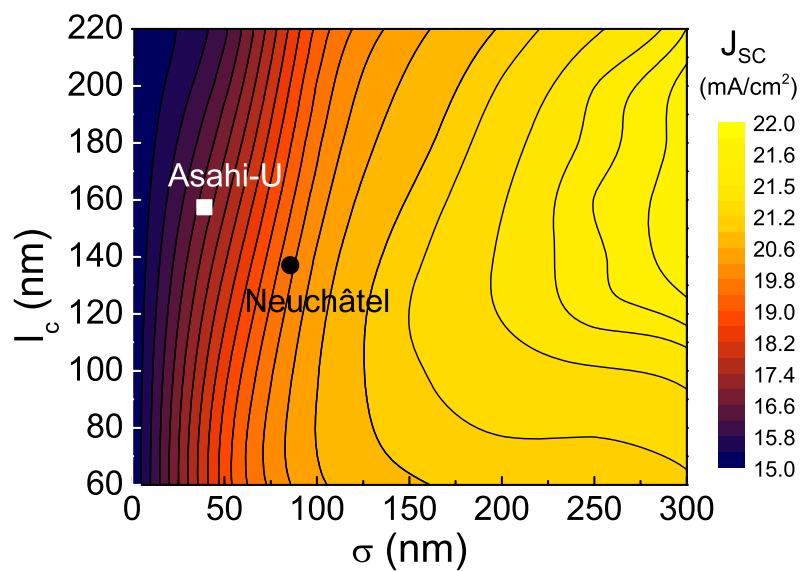

Fig. 5. (Color online) Short-circuit current density as a function of RMS height and correlation length. The two points denote the parameters of two common rough substrates.

higher for $2 \mathrm{D}$ rough substrates. The difference can be estimated by calculating the Lambertian light-trapping limit (for a single wavelength, the light path enhancement is $\pi n$ for $1 \mathrm{D}$, and $4 n^{2}$ for $\left.2 \mathrm{D}[2,17]\right)$. The short-circuit current density for a $1 \mu \mathrm{m}$ thick c-Si layer is calculated to be $22.7 \mathrm{~mA} / \mathrm{cm}^{2}$ with a $1 \mathrm{D}$, and $28.7 \mathrm{~mA} / \mathrm{cm}^{2}$ with a 2D Lambertian scatterer. The values reported in Fig. 5 are close to the 1D Lambertian limit. Thus, we expect an optimized $2 \mathrm{D}$ roughness to yield a $J_{\mathrm{sc}}$ about $6 \mathrm{~mA} / \mathrm{cm}^{2}$ higher than for the optimized 1D roughness.

Since the values of $J_{\mathrm{sc}}$ depend on several factors (material absorption, electronic transport, assumptions for solar spectrum), the effect of roughness and comparison with real thin-film solar cells should better be understood in terms of a relative enhancement. Our results for an optimized 1D substrate lead to a relative increase of $J_{\text {sc }}$ by $44 \%$. In [24,25], for example, the short-circuit current density of $\mu c$-Si:H solar cells of about $1 \mu \mathrm{m}$ thickness on TCO substrates with various degrees of roughness is determined, and the reported relative increase of $J_{\mathrm{sc}}$ is $+33 \%$ and $+21 \%$, respectively. Thus, in these cases, the calculated $J_{\mathrm{sc}}$ enhancement of a substrate with optimized 1D roughness is larger than for real solar cells with rough $2 \mathrm{D}$ substrates.

In conclusion, we performed a theoretical study of a randomly rough Gaussian interface, and we showed that, unexpectedly, this simple 1D model can be used to describe with high accuracy the optical properties of existing $2 \mathrm{D}$ rough interfaces. We then applied this model to the study of thin-film c-Si solar cells, and quantified the increase of short-circuit current density by the rough interface as a function of texture height and lateral correlation length. Moreover, we showed that light absorption close to the Lambertian limit can be obtained by the simultaneous optimization of the two statistical parameters. Given the simplicity of this model and its implementation, these results open the path toward the analysis of more complex architectures for light trapping in thin-film solar cells.

This work was supported by the EU through Marie Curie Action FP7-PEOPLE-2010-ITN Project No. 264687 "PROPHET" and Fondazione Cariplo project 2010-0523 "Nanophotonics for thin-film photovoltaics".

\section{References}

1. R. Brendel, Thin-Film Crystalline Silicon Solar Cells (Wiley-VCH, 2003).

2. E. Yablonovitch, J. Opt. Soc. Am. 72, 899 (1982).

3. S. Fahr, C. Rockstuhl, and F. Lederer, Appl. Phys. Lett. 92, 171114 (2008).

4. D. Dominé, F.-J. Haug, C. Battaglia, and C. Ballif, J. Appl. Phys. 107, 044504 (2010).

5. C. Rockstuhl, S. Fahr, K. Bittkau, T. Beckers, R. Carius, F.-J. Haug, T. Söderström, C. Ballif, and F. Lederer, Opt. Express 18, A335 (2010).

6. X. Sheng, S. G. Johnson, J. Michel, and L. C. Kimerling, Opt. Express 19, A841 (2011).

7. S. Fahr, T. Kirchartz, C. Rockstuhl, and F. Lederer, Opt. Express 19, A865 (2011).

8. F. Lederer, S. Fahr, C. Rockstuhl, and T. Kirchartz, in $M R S$ Proceedings (2012), Vol. 1391.

9. I. Simonsen, Eur. Phys. J. Special Topics 181, 1 (2010).

10. A. A. Maradudin and T. Michel, J. Stat. Phys. 58, 485 (1990).

11. V. Freilikher, E. Kanzieper, and A. Maradudin, Phys. Rep. 288, 127 (1997).

12. D. M. Whittaker and I. S. Culshaw, Phys. Rev. B 60, 2610 (1999).

13. M. Liscidini, D. Gerace, L. C. Andreani, and J. E. Sipe, Phys. Rev. B 77, 035324 (2008).

14. E. D. Palik, Handbook of Optical Constants of Solids (Academic, 1985).

15. V. Depauw, Y. Qiu, K. Van Nieuwenhuysen, I. Gordon, and J. Poortmans, Prog. Photovoltaics 19, 844 (2011).

16. A. Chutinan and S. John, Phys. Rev. A 78, 023825 (2008).

17. Z. Yu, A. Raman, and S. Fan, Opt. Express 18, A366 (2010).

18. C. Battaglia, C.-M. Hsu, K. Söderström, J. Escarré, F.-J. Haug, M. Charrière, M. Boccard, M. Despeisse, D. T. L. Alexander, M. Cantoni, Y. Cui, and C. Ballif, ACS Nano 6, 2790 (2012).

19. A. Oskooi, P. A. Favuzzi, Y. Tanaka, H. Shigeta, Y. Kawakami, and S. Noda, Appl. Phys. Lett. 100, 181110 (2012).

20. J. Nelson, The Physics of Solar Cells (Imperial College, 2003).

21. A. Bozzola, M. Liscidini, and L. C. Andreani, Opt. Express 20, A224 (2012).

22. D. Gerace and L. C. Andreani, Opt. Lett. 29, 1897 (2004).

23. S. Hughes, L. Ramunno, J. F. Young, and J. E. Sipe, Phys. Rev. Lett. 94, 033903 (2005).

24. M. Berginski, J. Hüpkes, M. Schulte, G. Schöpe, H. Stiebig, B. Rech, and M. Wuttig, J. Appl. Phys. 101, 074903 (2007).

25. H. Sai, H. Fujiwara, M. Kondo, and Y. Kanamori, Appl. Phys. Lett. 93, 143501 (2008). 